\title{
ORIGINAL RESEARCH \\ Recovery of Ophthalmoplegia after Endovascular Treatment of Intracranial Aneurysms
}

V. Panagiotopoulos

S.C. Ladd

E. Gizewski

S. Asgari

E.I. Sandalcioglu

M. Forsting

I. Wanke

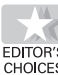

BACKGROUND AND PURPOSE: Recovery of aneurysm induced CNP after endosaccular coiling has been reported in the literature. The aim of this study was to assess in detail the parameters that affect the outcome after endovascular treatment of ophthalmoplegic aneurysms due CNP.

MATERIALS AND METHODS: Between November 1999 and March 2008, 30 consecutive patients (8 men, 22 women; mean age, 54.9 years) presenting with CNP underwent endosaccular coiling with or without additional use of stents in the parent artery. Subarachnoid hemorrhage was present in 10 patients, whereas 20 patients had unruptured aneurysms. The mean size of the aneurysms was $10 \mathrm{~mm}$. Initial CNP was complete in 11 patients and partial in 19. Mean follow-up after coiling was 19 months.

RESULTS: The mean interval between the onset of CNP and aneurysm embolization was 48 days Fifteen patients $(50 \%)$ had complete recovery of oculomotor function, 12 had incomplete recovery $(40 \%)$, and $3(10 \%)$ remained unchanged after treatment. In 4 aneurysms (13.3\%), 1 additional embolization was performed, whereas in 4 other aneurysms, 2 additional embolization procedures were necessary. Procedure-related permanent morbidity occurred in 2 patients (6.6\%)

CONCLUSIONS: Endosaccular coiling is an effective and safe method for the treatment of ophthalmoplegic aneurysms. Age, neck size, and time of treatment do not seem to constitute prognostic factors with respect to CNP recovery, though patients with small aneurysms, unruptured status, and/or location in the posterior circulation showed a tendency for better outcome. The degree of initial CNP was the only statistically significant prognostic factor concerning the final outcome, resulting in better recovery, in case of incomplete initial CNP.

ABBREVIATIONS: AchoA $=$ anterior choriod artery; Car.ophth $=$ carotid-ophthalmic artery; Cav $=$ cavernous segment of the ICA; CN = cranial nerve; CNP = third, fourth, and sixth cranial nerve paresis; $\mathrm{DSA}=$ digital subtracted angiography; ICA = internal carotid artery; $\mathrm{MCA}=$ middle cerebral artery; MRA = MR angiography; $\mathrm{mRS}=$ modified Rankin Scale; ONP = oculomotor nerve paresis; PcomA = posterior communicating artery; paraophth = paraophthalmic segment; Post. circ $=$ posterior cerebral circulation

$\mathbf{0}$ phthalmoplegia due to CNP is a clinical sign that may be associated with intracranial aneurysms and, in this situation, is caused by either enlargement of an unruptured aneurysm or rupture of the aneurysm sac. The most common aneurysm locations related to isolated unilateral oculomotor nerve paresis are the ICA-PcomA (89\%); the internal carotid cavernous segment and bifurcation (6.2\%); the basilar tip (3.4\%); and, extremely rarely, the anterior communicating artery, the anterior cerebral artery, and the middle cerebral artery, according to surgical series. ${ }^{1}$

Oculomotor nerve paresis has been reported to occur in $\leq 34 \%-56 \%$ of patients after rupture of aneurysms located in the ICA-PcomA. ${ }^{2-4}$ In another series, the incidence associated with ruptured and unruptured PcomAs was $16 \%{ }^{1}$

Although endovascular coiling is a less invasive alternative method to microsurgical clipping because it is associated with lower complication rates, its efficacy concerning recovery of ophthalmoplegia has not yet been adequately assessed, to our

Received April 10, 2010; accepted after revision July 4

From the Departments of Diagnostic and Interventional Radiology and Neuroradiology (V.P., S.C.L., E.G., M.F., I.W.) and Neurosurgery (S.A., E.I.S.), University Hospital of Essen, Essen, Germany; Departments of Neurosurgery and Interventional Neuroradiology (V.P.), University Hospital of Patras, Greece; and Department of Neuroradiology (I.W.), Klinik Hirslanden, Zurich, Switzerland.

Please address correspondence to Isabel Wanke, MD, Department of Diagnostic and Interventional Radiology and Neuroradiology, University Hospital Essen, Hufelandstr 55, D-45122, Essen, Germany; e-mail: isabel.wanke@uni-duisburg-essen.de

DOl 10.3174/ajnr.A2281

knowledge, due to the limited number of patients included in series reported in the literature. A more detailed insight into the influence of patient and treatment parameters could assist in the decision for optimal patient selection for endovascular treatment as well as for choosing the best time for the treatment.

Thus, the purpose of our study was to evaluate which parameters influence the outcome after endosaccular coiling in patients presenting with CNP in a larger number of patients.

\section{Materials and Methods}

\section{Patients}

Between November 1999 and March 2008, thirty consecutive patients presenting with ophthalmoplegia due to CNP in the department of interventional neuroradiology underwent endovascular treatment by means of endosaccular coiling with or without additional use of stents in the parent artery. Their medical records and angiographic studies were analyzed retrospectively.

\section{Definitions, Clinical Evaluation, and Follow-Up}

Preinterventional CNP was categorized as complete in case of diplopia, ptosis, ophthalmoplegia, and pupillary dysfunction and as incomplete in cases of similar symptoms associated with preserved partial extraocular movement in upward, medial, and downward gazes or pupillary sparing. 
The postoperative evolution of CNP was classified as unchanged, improved (incomplete recovery), or complete recovery.

"Complete recovery" was considered in case of complete resolution of diplopia; resolution of ptosis; recovery of the full range of movement in medial, downward, and upward gaze; and partial or complete recovery of pupillary reaction. In any case of only partial improvement, CNP recovery was considered to be incomplete.

The interval between primary onset of CNP and the day of intervention was recorded.

Neuro-ophthalmologic assessment, conducted by a neuro-ophthalmologist, was scheduled before embolization; at discharge; after 3,6 , and 12 months; and then annually or in case of recurrent symptoms. The "time to CNP recovery" was defined as the interval from the treatment day to the day of a normal extraocular movement assessed either at discharge or at the scheduled neuro-ophthalmologic follow-up.

Assessment of the clinical outcome, by using the mRS for the degree of disability, was performed at patient admission and discharge and was scheduled at 6 and 18 months after the intervention.

Angiographic follow-up was scheduled at 6 months after treatment, including DSA and MRA. In case no recanalization was observed, a further angiographic control study with MRA was performed 12 months later and annually thereafter. In case of recanalization, individual evaluation of each case was performed, considering the clinical symptoms and the degree of potential morbidity.

The degree of intrasaccular embolization of the aneurysm lumen by the coils, on postoperative and on control imaging (DSA and/or MRA), was assessed by use of the modified 3-point Raymond scale (Raymond $1=$ complete obliteration of aneurysm including the neck, Raymond $2=$ contrast filling the neck of the aneurysm without opacification of the aneurysm sac, and Raymond $3=$ contrast filling of the sac of the aneurysm). ${ }^{5}$ Angiographic recurrences that needed to be retreated have been noted separately.

Other parameters that were noted separately were the following: patient age, duration of CNP before intervention (time interval), the grade of preinterventional CNP, the aneurysm location, the size of the aneurysm, the size of the aneurysm neck, and the presence or absence of aneurysm rupture before intervention. These parameters were then related to the final clinical outcome (unchanged, improved, or complete recovery).

\section{Statistical Analysis}

Due to the limited number of individuals, results are presented mainly in a descriptive manner. Differences in the outcome for different groups were analyzed for statistical significance by use of the $\chi^{2}$ test or the Fisher exact test.

\section{Results}

\section{Clinical Symptoms and Findings}

Among the 30 patients ( 8 men, 22 women) who were included in the study, 11 initially presented with complete and 19 with incomplete ocular motor nerve (third, fourth, and sixth) paresis. Twenty were admitted without aneurysm rupture, whereas 10 were admitted with subarachnoid hemorrhage due to aneurysm rupture. The mean age of the patients was 53 years (range, 14-75 years). Ten aneurysms were located at the PcomA, 11 in the carotid cavernous segment, 4 in the supra-/ paraophthalmic carotid segment, and 5 in the basilar, poste-

\begin{tabular}{lc}
\hline \multicolumn{2}{l}{ Table 1: Patient demographics and aneurysm characteristics } \\
\hline Characteristic/Demographics & Data \\
\hline Patients (No.) & 30 \\
Age (mean) (yr) & 53 (range, 14-75) \\
Sex & 8 \\
$\quad$ Male & 22 \\
$\quad$ Female & \\
ONP (at presentation) & 19 \\
$\quad$ Incomplete & 11 \\
Complete & \\
Aneurysm location & 11 \\
$\quad$ ICA-cavernous & 10 \\
ICA-PcomA & 4 \\
ICA-supraophthalmic & 5 \\
$\quad$ Posterior circulation & 10 (range, 2-27). \\
Aneurysm size (mean) (mm) & 10 \\
Rupture & 20 \\
$\quad$ Yes & 48 (range, 1-240) \\
$\quad$ No & 69 (range, 1-300) \\
Time to treatment (mean) (days) &
\end{tabular}

rior cerebral, or superior cerebellar arteries. The mean size of the aneurysms was $10 \mathrm{~mm}$ (range, $2-27 \mathrm{~mm}$ ). Table 1 gives an overview of patient demographics and assessed parameters.

\section{Treatment and Outcome}

The mean interval between the onset of CNP and aneurysm embolization was 48 days (range, 1-240 days). The mean postinterventional follow-up was 19 months (range, 0.2-73 months). Overall, 15 patients (50\%) experienced complete recovery of ocular motor function, 12 had incomplete recovery (40\%), and $3(10 \%)$ remained unchanged after treatment. The mean interval between the treatment and recovery of CNP was 69 days (range, 1-300 days).

No statistically significant correlation could be found between age and final degree of $\mathrm{CNP}$ recovery. Also the diameter of the neck did not significantly contribute to different outcomes of CNP recovery. No relationship could be found between $\mathrm{CNP}$ recovery and the time interval (duration of CNP before intervention).

According to aneurysm location, complete resolution of $\mathrm{CNP}$ after endovascular treatment was accomplished in 54.5\% $(6 / 11)$ of the aneurysms located in the carotid cavernous segment, in $50 \%(2 / 4)$ of the aneurysms located in the supra-/ paraophthalmic carotid segment, and in $80 \%(4 / 5)$ of the aneurysms of the posterior circulation, whereas complete resolution occurred in only $30 \%$ (3/10) of the aneurysms located in the PcomA. Table 2 illustrates in detail the grade of $\mathrm{CNP}$ recovery with respect to aneurysm location.

Smaller aneurysms $(\leq 10 \mathrm{~mm})$ showed a higher tendency for better CNP recovery (complete recovery, 11/20; 55\%) compared with larger aneurysms $(>10 \mathrm{~mm})$ (complete recovery, $4 / 10 ; 40 \%$ ) (Fig 1). More patients achieved a complete recovery of CNP in cases of unruptured aneurysms $(12 / 18 ; 67 \%)$ than in cases of ruptured aneurysms (3/12, 25\%) (Fig 2); nevertheless, both of these differences were not statistically significant.

The degree of preinterventional CNP (ie, complete or partial) was the only parameter that affected significantly $(P=$ $.009)$ the recovery degree of ophthalmoplegia in our patients: Thirteen of 19 patients $(68.4 \%)$ who initially presented with 


\begin{tabular}{lcccc}
\hline \multicolumn{4}{l}{ Table 2: Evolution of ONP after endovascular treatment according to aneurysm location } & \\
\hline & $\begin{array}{c}\text { Complete ONP } \\
\text { Recovery } \\
\text { (Postintervention) }\end{array}$ & $\begin{array}{c}\text { Incomplete ONP } \\
\text { Recovery } \\
\text { (Postintervention) }\end{array}$ & $\begin{array}{c}\text { No Improvement } \\
\text { (Postintervention) }\end{array}$ & $\begin{array}{c}\text { Total No. } \\
\text { of Patients }\end{array}$ \\
\hline ICA-PcomA & $3 / 10(30 \%)$ & $6 / 10(60 \%)$ & $1 / 10(10 \%)$ & 10 \\
ICA-cavernous & $6 / 11(54.5 \%)$ & $4 / 11(36.4 \%)$ & $1 / 11(9.1 \%)$ & 11 \\
ICA-supraophthalmic & $2 / 4(50 \%)$ & $1 / 4(25 \%)$ & $1 / 4(25 \%)$ & 4 \\
Posterior circulation & $4 / 5(80 \%)$ & $1 / 5(20 \%)$ & $0 / 5(0 \%)$ & 5 \\
\hline
\end{tabular}

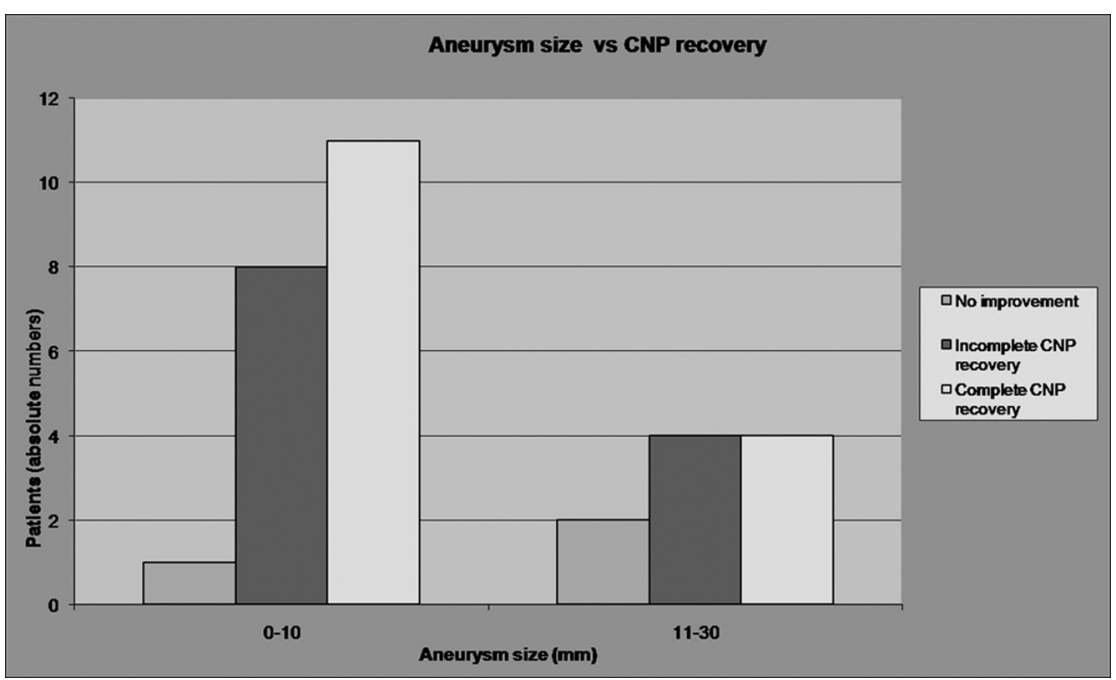

Fig 1. Graph shows that smaller aneurysms ( $\leq 10 \mathrm{~mm}$ ) have a higher but not statistically significant tendency for better CNP recovery (complete recovery, 11/20; 55\%) compared with larger aneurysms (>10 mm) (complete recovery, 4/10; 40\%).

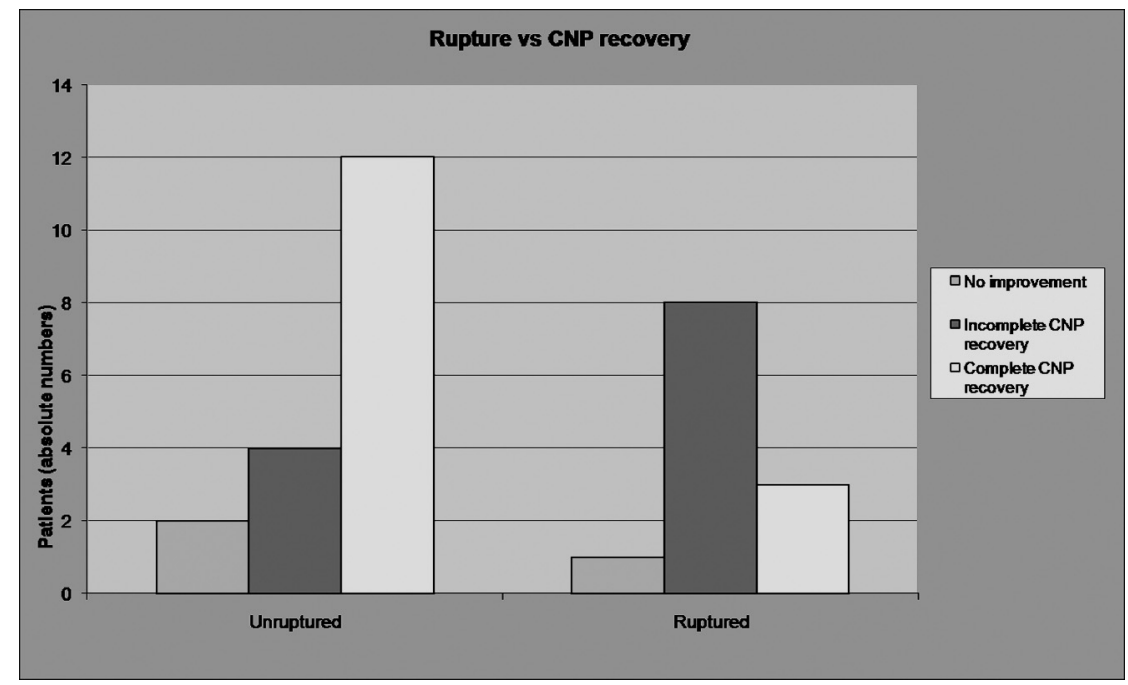

Fig 2. Graph shows that more patients achieve a complete recovery of CNP in cases of unruptured aneurysms $(12 / 18,67 \%)$ than in cases of ruptured aneurysms $(3 / 12,25 \%)$. The difference is not statistically significant.

incomplete CNP recovered completely, whereas 6/19 patients $(31.6 \%)$ recovered incompletely $(n=4)$ or had no improvement $(n=2)$ after endovascular treatment. On the other hand, only $2 / 11(18.2 \%)$ of those patients who initially presented with complete CNP recovered fully, whereas 9/11 of those patients $(81.8 \%)$ recovered incompletely $(n=8)$ or had no improvement $(n=1)$ after coiling (Fig 3$)$. Table 3 illustrates statistical tests and the $P$ values concerning each variable.

A disadvantage of endovascular treatment is the potential recanalization of the aneurysm sac. According to our results, in 4 aneurysms, 1 retreatment had to be performed, whereas in
4 other aneurysms, 2 retreatment procedures for each aneurysm were necessary due to recanalization, in a mean follow-up of 19 months. The mean size of the recanalized aneurysms was 13 $\mathrm{mm}$ (range, 7-20 $\mathrm{mm}$ ), and 6/8 of these recanalized aneurysms had a neck width of $>4 \mathrm{~mm}$. No procedure-related morbidity due to recoiling was added to our cohort.

\section{Complications}

Postembolization Clinical Morbidity. According to the mRS, 3 patients (3/30) experienced nondisabling (mRS 1-2) neurologic deficits, whereas an additional 3 patients (3/30) 


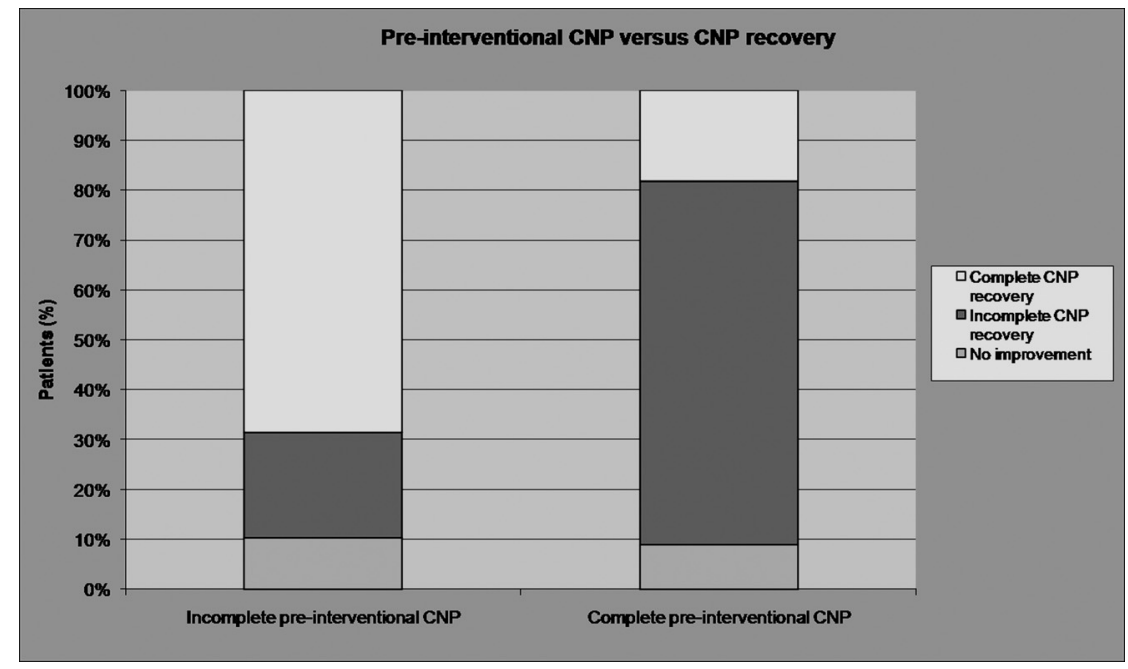

Fig 3. Graph shows that the degree of preinterventional CNP (ie, complete or partial) is the only parameter that affects significantly $(P=.009)$ the recovery of ophthalmoplegia. Patients with incomplete initial CNP have a statistically significant higher degree of CNP recovery.

\begin{tabular}{|c|c|c|}
\hline Parameter & Statistical Test & $\begin{array}{c}P \\
\text { Value }\end{array}$ \\
\hline$\overline{\text { Patient age }}$ & Spearman correlation & .157 \\
\hline Aneurysm neck size & Fisher exact test & .458 \\
\hline $\begin{array}{l}\text { Duration of CNP before intervention } \\
\text { (time interval) }\end{array}$ & Spearman correlation & .835 \\
\hline Aneurysm size & Spearman correlation & .547 \\
\hline Rupture of aneurysm & Fisher exact test & .082 \\
\hline Grade of preinterventional CNP & Fisher exact test & .009 \\
\hline Degree of aneurysm embolization & Fisher exact test & .391 \\
\hline Aneurysm location & Fisher exact test & .588 \\
\hline
\end{tabular}

experienced disabling $(\mathrm{mRS} \geq 3$ ) neurologic deficits immediately after all the initial embolization procedures. Nondisabling neurologic deficits included 1 case of speech disturbance, 1 case of trigeminal neuralgia, and 1 case of third and sixth nerve palsy worsening, whereas disabling neurologic deficits included 1 case of global aphasia and 2 cases of hemiparesis with muscle strength 4/5 (movement possible against some resistance by the examiner; 1 case of hemiparesis was followed by speech disturbance, whereas the other was followed by seventh nerve paresis).

Among these 6 (20\%) patients with emerging neurologic deficits, the underlying causes were the following: 1 ) a temporary thrombotic occlusion of the middle cerebral artery ( 1 case of hemiparesis followed by speech disturbance), 2) a mass effect on the gasserian ganglion due to size increase after stent placement and coiling of a 22-mm cavernous aneurysm ( 1 case of trigeminal neuralgia), 3) mild pressure on the cavernous part of the third and sixth nerves after coiling of a $19-\mathrm{mm}$ cavernous aneurysm ( 1 case of third and sixth nerve palsy worsening), and 4-6 in the cases of speech disturbance as well as in 2 cases of neurologic deficits ( 1 case of global aphasia and 1 case of hemiparesis followed by seventh nerve paresis), no intraprocedural complication or underlying cause was identified.

Procedure-Related Clinical Morbidity at Follow-Up. At a mean follow-up of 19 months (range, 0.2-73 months), the initially nondisabling neurologic deficits in 2 patients ( 1 case of trigeminal neuralgia and 1 case of third and sixth nerve palsy worsening) showed complete resolution. The third patient with a speech disturbance showed partial improvement.

Concerning the initially disabling neurologic deficits, $2 \mathrm{pa}-$ tients ( 1 case of global aphasia and 1 case of hemiparesis followed by seventh nerve paresis) showed complete resolution of their symptoms, whereas in a third patient the hemiparesis followed by speech disturbance did not improve.

Recoiling procedures did not add clinical morbidity to our cohort.

At the end of the follow-up, nondisabling (mRS 1-2) embolization-related clinical morbidity was seen in 1 patient $(3.3 \%)$, whereas disabling ( $\mathrm{mRS} \geq 3$ ) embolization-related clinical morbidity occurred in 1 patient $(3.3 \%)$ (Table 4$)$. The total permanent morbidity in our patients was $6.6 \%(2 / 30)$.

\section{Discussion}

Several pathogenetic mechanisms have been described in the literature concerning third nerve paresis, such as direct compression by an enlarged aneurysmal sac within the suprasellar cistern, ${ }^{6}$ pulsatility inside the aneurysm, ${ }^{7}$ or an irritating effect of subarachnoid blood. ${ }^{8}$ Mydriasis can be caused by an ICAPcomA aneurysm compressing the oculomotor nerve superomedially and, more specifically, the dorsomedially located iridoconstrictor fibers. ${ }^{9}$ Compression of the pain sensory afferent fibers, which are recruited from the ophthalmic division of the trigeminal nerve and which lie within the periphery of the oculomotor nerve, causes ipsilateral orbitofacial pain. ${ }^{10}$ Compressive lesions of the cavernous sinus may lead to localized oculomotor or abducens $\mathrm{CN}$ dysfunction and Horner syndrome. ${ }^{11}$ Evolution of ocular motor nerve (third, fourth, and sixth) paresis after endovascular therapy has been reported in the literature: Beyond sporadic reports with ONP resolution after coiling of PcomA aneurysms, ${ }^{12}$ a literature review concerning series including $\geq 3$ aneurysms revealed a mean rate of $46.6 \%$ complete recovery after endovascular treatment of $118(55 / 118)$ aneurysms that caused ophthalmoplegia (Table 5). ${ }^{7,9,13-21}$ Our results concerning endovascular treatment are very encouraging: In a mean follow-up of 19 


\begin{tabular}{lll} 
Postembolization Clinical Morbidity & \multicolumn{1}{c}{ Identified Cause } & (mean, 19 mo; range, $0.2-73$ mo) \\
\hline Speech disturbance & Not identified & Partial improvement \\
Trigeminal neuralgia & Compression of the gasserian ganglion & Complete resolution \\
Worsening of CNP of III and VI & Mild pressure on the cavernous part of nerves III and VI & Complete resolution \\
Global aphasia & Not identified & Complete resolution \\
Hemiparesis $(4 / 5)+$ speech disturbance & MCA temporary occlusion & No improvement \\
Hemiparesis $(4 / 5)+$ paresis of nerve VII & Not identified & Complete resolution \\
\hline
\end{tabular}

\begin{tabular}{|c|c|c|c|c|c|c|}
\hline Study & Patients & Location & $\begin{array}{l}\text { Time to Treatment } \\
\text { (mean) }\end{array}$ & $\begin{array}{l}\text { Complete } \\
\text { Recovery }\end{array}$ & $\begin{array}{l}\text { Incomplete } \\
\text { Recovery }\end{array}$ & $\begin{array}{c}\text { No } \\
\text { Improvement }\end{array}$ \\
\hline Birchall et al (1999) & 3 & PcomA & 2.3 days & $3(100 \%)$ & 0 & 0 \\
\hline Mavilio et al $(2000)^{14}$ & 6 & PcomA & 2 days to 6 mo (range) & $6(100 \%)$ & 0 & 0 \\
\hline Kazekawa et al $(2003)^{13}$ & 12 & PcomA-2/Cav-6/Car.ophth-2 & $13.7 \mathrm{mo}$ & $5(42 \%)$ & $4(33 \%)$ & $3(25 \%)$ \\
\hline Kim et al $(2003)^{9}$ & 7 & PcomA-2/Cav-3/Basilar-2 & $11 \mathrm{mo}$ & $2(29 \%)$ & $3(42 \%)$ & $2(29 \%)^{a}$ \\
\hline Stiebel-Kalish et al $(2003)^{17}$ & 11 & PcomA & 9 days & 0 & $11(100 \%)$ & 0 \\
\hline Chen et al $(2006)^{18}$ & 6 & PcomA & 5.5 days & $4(33 \%)$ & $2(67 \%)$ & 0 \\
\hline Ahn et al $(2006)^{21}$ & 10 & PcomA & Unknown & $6(60 \%)$ & $3(30 \%)$ & $1(10 \%)$ \\
\hline Mansour et al $(2007)^{15}$ & 11 & PcomA-6/Cav-5 & 10 weeks & $7(64 \%)$ & $4(36 \%)^{b}$ & \\
\hline Hanse et al $(2008)^{16}$ & 21 & PcomA & 1 to $>14$ days (range) & $8(38.1 \%)$ & $11(52.4 \%)$ & $2(9.5 \%)$ \\
\hline Kassis et al $(2010)^{20}$ & 20 & PcomA & 71 days & $7(35 \%)$ & $12(60 \%)$ & $1(5 \%)$ \\
\hline Santillan et al $(2010)^{19}$ & 11 & PcomA & $\leq 7$ days & $7(64 \%)$ & $2(18 \%)$ & $2(18 \%)$ \\
\hline Current study & 30 & $\begin{array}{l}\text { PcomA-10/Cav-11/Post.circ-5/ } \\
\text { paraophth-4 }\end{array}$ & 48 days & $15(50 \%)$ & $12(40 \%)$ & $3(10 \%)$ \\
\hline
\end{tabular}

a No improvement or worsening.

${ }^{\mathrm{b}}$ Incomplete recovery or no improvement.

months (range, 0.2-73 months), complete resolution of ocular motor nerve (third, fourth, and sixth) paresis occurred in $50 \%$ of our patients, whereas $40 \%$ recovered incompletely and only $10 \%$ showed no improvement.

These results seem to be in the upper range or, in some cases, superior to the results previously reported in endovascular series. It seems probable that coiling eliminates the pulsatility inside the aneurysm sac and, therefore, the "hammer effect" on the nerve. This mechanism offers the opportunity for the affected nerve to recover partially or completely in most of the cases treated endovascularly. Nevertheless, in patients with long-standing and severe third nerve paralysis, aberrant regeneration with misdirection of fibers in a noncoordinated way may occur, leading to poor clinical outcome. In contrast, complete recovery with good clinical outcome is quite common in isolated sixth or fourth nerve palsies because these nerves innervate only 1 muscle and the phenomenon of aberrant disorganized regeneration does not occur. In case of ruptured aneurysms, coiling protects additionally from further potential rebleeding and allows resorption of the subarachnoid blood, thus resolving gradually its irritating effect on the nerve. In this respect, endovascular coiling seems to be an alternative minimally invasive method to neurosurgical clipping.

The positive effect of early treatment on final ONP recovery has been shown in several studies concerning endovascular treatment of ophthalmoplegic aneurysms ${ }^{7,9,13,15}$ : Kazekawa et $\mathrm{al}^{13}$ demonstrated that the group with resolution or improvement of symptoms had a shorter duration of symptoms before coiling $(P<.05)$. Mansour et $\mathrm{al}^{15}$ reported that symptoms resolved in $75 \%$ of patients presenting with them for $<1$ month, whereas the rate dropped to $33.3 \%$ in cases in which symptoms lasted for $>4$ weeks. Unlike these findings, in our study, the interval time between CNP and the intervention did not affect the degree of postintervention resolution of CNP. This finding was in accordance with previous series concerning coiling of aneurysms that caused ophthalmoplegia. ${ }^{16,17}$

As reported in previous studies, aneurysm size had no predictive value concerning recovery of ophthalmoplegia after endovascular treatment. ${ }^{13,16,18}$ Nevertheless in a series by Mansour et $\mathrm{al}^{15}{ }^{15}$ the complete resolution rate was $71.4 \%$ for aneurysms $<15 \mathrm{~mm}$ and $50 \%$ for aneurysms $>15 \mathrm{~mm}$. According to our results, though smaller aneurysms ( $\leq 10 \mathrm{~mm})$ showed a higher tendency for better CNP recovery, the difference in the outcome between sizes $<10 \mathrm{~mm}$ and $\geq 10 \mathrm{~mm}$ was not statistically significant (Fig 1); thus, aneurysm size was not considered to have a predictive impact.

Although Mansour et $\mathrm{al}^{15}$ reported a $42.8 \%$ complete recovery rate of unruptured aneurysms treated endovascularly and a rate for ruptured aneurysms of $100 \%$, Chen et al, ${ }^{18}$ who compared coiling versus clipping as a treatment of ONP aneurysms, demonstrated no effect of the presence of subarachnoid hemorrhage on recovery of ONP. In our study, more patients achieved a complete recovery of CNP in cases of unruptured aneurysms $(6 / 18,67 \%)$ than in cases of ruptured aneurysms $(3 / 12,25 \%)$; nevertheless, this tendency did not reach statistical significance.

Concerning the outcome of CNP after endovascular treatment according to location, Kim et $\mathrm{al}^{9}$ reported a higher tendency concerning CNP improvement or resolution for intradural than for extradural aneurysms, with a rate of $89 \%$ and $17 \%$, respectively. This tendency could not be reproduced in our series. According to our results, as illustrated in Table 2, the highest tendency for complete CNP recovery occurred in 

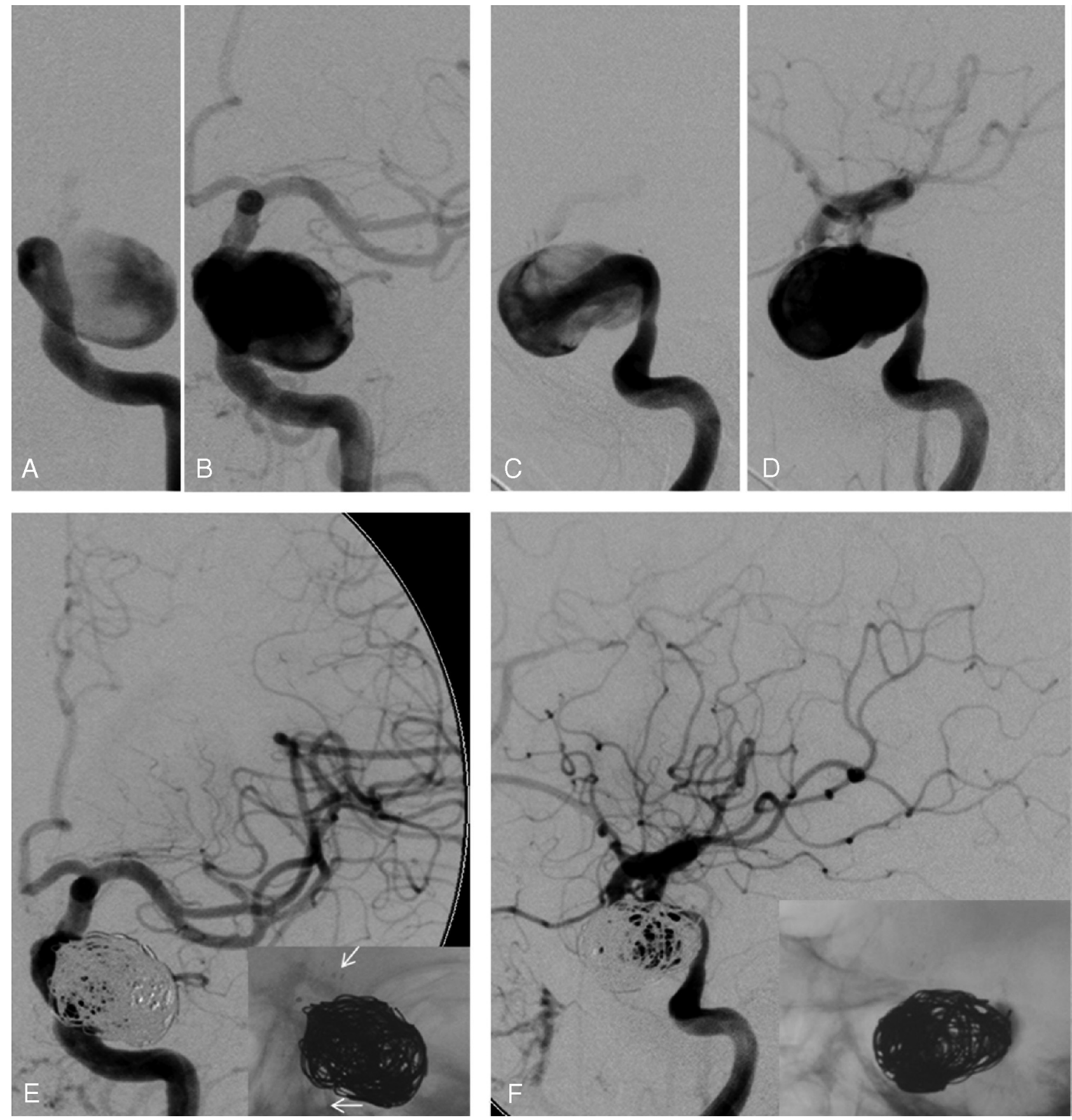

Fig 4. Unruptured cavernous aneurysm of the left ICA causing partial sixth cranial nerve paresis for 2 months. Anteroposterior ( $A$ and $B$, early and middle arterial phase) and lateral arteriograms $(C$ and $D$, early and middle arterial phase) before intervention. Anteroposterior $(E)$ and lateral arteriograms $(F)$ after stent-supported coiling. The white arrows in the insert of the anteroposterior arteriogram $(E)$ show the stent struts.

aneurysms located in the posterior circulation $(4 / 5,80 \%)$, whereas the lowest tendency for complete resolution occurred in aneurysms located in the ICA-PcomA (3/10, 30\%).

According to our results, the degree of initial CNP (ie, complete or partial) was the only parameter that affected significantly $(P=.009)$ the recovery degree of nerve function after endovascular treatment in our patients: The percentage of complete CNP resolution was 68.4\% (13/19 patients) after endovascular treatment in patients initially presenting with incomplete $\mathrm{CNP}$, whereas complete recovery occurred in only $18.2 \%$ (2/11 patients) of those initially presenting with complete CNP (Fig 3). Figure 4 illustrates a case of a patient with a partial sixth nerve paresis for 2 months due to an unruptured cavernous aneurysm. The paresis recovered fully after endovascular treatment with stent-supported coiling. As previously reported in the literature, the degree of initial CNP plays a significant role in the final outcome either after endovascular or surgical treatment.

Mansour et $\mathrm{al}^{15}$ reported $100 \%$ resolution of symptoms after coiling in patients presenting initially with partial CNP and $50 \%$ resolution in patients presenting initially with complete CNP. Hanse et a ${ }^{16}$ showed that initial partial palsy of $\mathrm{CN}$ III was the only predictor of complete recovery at follow-up. In this series, $83.3 \%$ of patients who initially presented with 


\begin{tabular}{|c|c|c|c|c|c|c|}
\hline Study & Patients & Location & $\begin{array}{l}\text { Time to Treatment } \\
\text { (mean) }\end{array}$ & $\begin{array}{l}\text { Complete } \\
\text { Recovery }\end{array}$ & $\begin{array}{l}\text { Incomplete } \\
\text { Recovery }\end{array}$ & $\begin{array}{c}\text { No } \\
\text { Improvement }\end{array}$ \\
\hline Literature review by Leivo et al (before 1996) & 283 & PcomA & Unknown & $117(41 \%)$ & $147(52 \%)$ & $19(7 \%)$ \\
\hline Leivo et al $(1996)^{1}$ & 28 & PcomA & 0 to $>30$ days & $16(57 \%)$ & Unknown & Unknown \\
\hline Yanaka et al $(2003)^{22}$ & $14^{\mathrm{a}}$ & PcomA-11/AchoA-2/basilar-1 & 17.3 days & $7(50 \%)$ & $6(43 \%)$ & $1(7 \%)$ \\
\hline Chen et al $(2006)^{18}$ & 7 & PcomA & $1-90$ days & $6(85.7 \%)$ & $1(14.7 \%)$ & 0 \\
\hline Ahn et al $(2006)^{21}$ & 7 & PcomA & Unknown & $3(42.9 \%)$ & $4(57.1 \%)$ & 0 \\
\hline
\end{tabular}

a Patients who underwent surgery.

incomplete palsy of CN III recovered completely, whereas only $20 \%$ of the patients initially presenting with complete palsy of CN III recovered completely. In a comparison study (coiling versus clipping for treatment of ophthalmoplegic aneurysms), Chen et $\mathrm{al}^{18}$ showed that the degree of oculomotor nerve paresis (ie, complete or partial) affected recovery of nerve function regardless of treatment method: Partial oculomotor nerve paresis promises better recovery after either clipping or coiling of a PcomA aneurysm.

Surgical treatment, by clipping the aneurysm neck, relieves the mass effect and offers adequate decompression of the nerve. In the literature, a mean rate of $44 \%$ complete recovery has been reported after surgical treatment of 339 (149/339) aneurysms that caused ophthalmoplegia (Table 6). ${ }^{1,18,21,22}$ Most of the aneurysms were located in the PcomA, whereas only 1 was located in the posterior circulation. The time interval from onset of oculomotor nerve paresis to treatment seems to be of critical importance: In a review performed by Leivo et al, ${ }^{1}$ the mean rate of $41 \%$ complete ONP recovery after clipping rose to $64 \%$ in patients undergoing surgery within 2 weeks, whereas the number diminished significantly in patients undergoing surgery later, within $14-30$ days (30\%) and after 1 month (14\%). Leivo et al reported a mean rate of 57\% complete ONP recovery in their own patients, which rose to $80 \%$ in patients undergoing surgery within 1 week. In another series by Yanaka et al, ${ }^{22}$ immediate surgical treatment (within 5 days from the onset of oculomotor palsy) offered the best rates for recovery of neural function. Although our study does not compare endovascular and surgical treatment of ophthalmoplegic aneurysms, our results $(50 \%$ complete resolution of CNP and $40 \%$ incomplete recovery) seem to be comparable with, or in some cases superior to, surgical outcomes reported in the literature.

Considering the high degree of complete and partial CNP recovery in our study (90\%) combined with a low permanent morbidity rate $(6.6 \%)$, we conclude that coiling is an effective and safe method for the treatment of aneurysms presenting with ophthalmoplegia due to CNP.

\section{Conclusions}

Age, neck size, and early treatment do not seem to constitute prognostic factors with respect to CNP recovery. Patients with small aneurysms, unruptured status, or location in the posterior circulation showed a higher tendency for better outcome of ophthalmoplegia, but these parameters did not reach significance and need to be further evaluated in a larger series. The degree of initial CNP was the only statistically significant prognostic factor for the final degree of CNP recovery.

\section{References}

1. Leivo S, Hernesniemi J, Luukkonen M, et al. Early surgery improves the cure of aneurysm-induced oculomotor palsy. Surg Neurol 1996;45:430-34

2. Soni SR. Aneurysms of the posterior communicating artery and oculomotor paresis. J Neurol Neurosurg Psychiatry 1974;37:475-84

3. Hamilton JG, Falconer MA. Immediate and late results of surgery in cases of saccular intracranial aneurysms. J Neurosurg 1959;16:514-41

4. Paterson A. Direct surgery in the treatment of posterior communicating artery aneurysms. Lancet 1968;2:808-11

5. Raymond J, Guilbert F, Weill A, et al. Long-term angiographic recurrences after selective endovascular treatment of aneurysms with detachable coils. Stroke 2003;34:1398-403

6. Giombini S, Farraresi S, Plunchino F. Reversal of oculomotor disorders after intracranial aneurysm surgery. Acta Neurochir 1991;112:19-24

7. Birchall D, Khangure MS, McAuliffe W. Resolution of third nerve paresis after endovascular management of aneurysms of the posterior communicating artery. AJNR Am J Neuroradiol 1999;20:411-13

8. Hyland $\mathrm{HH}$, Barnett HJ. The pathogenesis of cranial nerve palsies associated with intracranial aneurysms. Proc R Soc Med 1954;47:141-46

9. Kim DJ, Kim DI, Lee SK, et al. Unruptured aneurysms with cranial nerve symptoms: efficacy of endosaccular Guglielmi detachable coil treatment. Korean J Radiol 2003;4:141-45

10. Lanzino G, Andreoli A, Tognetti F, et al. Orbital pain and unruptured carotidposterior communicating artery aneurysms: the role of sensory fibers of the third cranial nerve. Acta Neurochir (Wien) 1993;120:7-11

11. Brazis PW. Localization of lesions of the oculomotor nerve: recent concepts. Mayo Clin Proc 1991;66:1029-35

12. Inamasu J, Nakamura Y, Saito R, et al. Early resolution of third nerve palsy following endovascular treatment of a posterior communicating artery aneurysm. J Neuroophthalmol 2002;22:12-14

13. Kazekawa $\mathrm{K}$, Tsutsumi $\mathrm{M}$, Aikawa $\mathrm{H}$, et al. Internal carotid aneurysms presenting with mass effect symptoms of cranial nerve dysfunction: efficacy and imitations of endosaccular embolization with GDC. Radiat Med 2003; 21:80-85

14. Mavilio N, Pisani R, Rivano C, et al. Recovery of third nerve palsy after endovascular packing of internal carotid-posterior communicating artery aneurysms. Intervent Neuroradiol 2000;6:203-09

15. Mansour N, Kamel MH, Kelleher M, et al. Resolution of cranial nerve paresis after endovascular management of cerebral aneurysms. Surg Neurol 2007;68: 500-04

16. Hanse MC, Gerrits MC, van Rooij WJ, et al. Recovery of posterior communicating artery aneurysm-induced oculomotor palsy after coiling. AJNR Am J Neuroradiol 2008;29:988-90

17. Stiebel-Kalish H, Maimon S, Amsalem J, et al. Evolution of oculomotor nerve paresis after endovascular coiling of posterior communicating artery aneurysms: a neuro-ophthalmological perspective. Neurosurgery 2003;53: 1268-73

18. Chen PR, Amin-Hanjani S, Albuquerque FC, et al. Outcome of oculomotor nerve palsy from posterior communicating artery aneurysms: comparison of clipping and coiling. Neurosurgery 2006;58:1040-46

19. Santillan A, Zink WE, Knopman J, et al. Early endovascular management of oculomotor nerve palsy associated with posterior communicating artery aneurysms. Interv Neuroradiol 2010;16:17-21. Epub 2010 Mar 25

20. Kassis SZ, Jouanneau E, Tahon FB, et al. Recovery of third nerve palsy after endovascular treatment of posterior communicating artery aneurysms. World Neurosurg 2010;73:11-16

21. Ahn JY, Han IB, Yoon PH, et al. Clipping vs coiling of posterior communicating artery aneurysms with third nerve palsy. Neurology 2006;10;66:121-23

22. Yanaka K, Matsumaru Y, Mashiko R, et al. Small unruptured cerebral aneurysms presenting with oculomotor nerve palsy. Neurosurgery 2003;52: $553-57$ 